\title{
Generalized Ratio-Cum-Product Type Estimator of Finite Population Mean in Double Sampling for Stratification
}

\author{
Rajesh Tailor ${ }^{a}$, Hilal A. Lone ${ }^{1, a}$, Rajiv Pandey ${ }^{b}$ \\ ${ }^{a}$ School of Studies in Statistics, Vikram University, India; \\ ${ }^{b}$ Hemwati Nandan Bahuguna Garhwal University Uttarakhand, India
}

\begin{abstract}
This paper addressed the problem of estimation of finite population mean in double sampling for stratification. This paper proposed a generalized ratio-cum-product type estimator of population mean. The bias and mean square error of the proposed estimator has been obtained upto the first degree of approximation. A particular member of the proposed generalized estimator was identified and studied from a comparison point of view. It is observed that the identified particular estimator is more efficient than usual unbiased estimator and Ige and Tripathi (1987) estimators. An empirical study was conducted in support of the theoretical findings.
\end{abstract}

Keywords: mean square error, bias, percent relative efficiency, finite population mean, double sampling for stratification

\section{Introduction}

Cochran (1940) and Robson (1957) envisaged classical ratio and product estimators which were studied in case of double sampling for stratification by Ige and Tripathi (1987). Singh (1967) used information on population mean of two auxiliary variates and proposed ratio-cum-product type estimators for population mean in simple random sampling. Later Koyuncu and Kadilar (2009) studied Singh (1967) ratio-cum-product type estimators in stratified random sampling. Singh (1967) and Koyuncu and Kadilar (2009) motivated authors to study ratio-cum-product type estimators in case of double sampling for stratification.

Singh and Vishwakarma (2007) discussed a general procedure for estimating the populations mean using double sampling for stratification. Tailor and Lone (2014) proposed a ratio-cum-product type estimator using linear combination of ratio and product type estimators in double sampling for stratification. Tailor et al. (2014) proposed ratio and product type exponential estimators of population mean in double sampling for stratification.

Let us consider a finite population $U=\left(U_{1}, U_{2}, \ldots, U_{N}\right)$ of size $N$ in which strata weight $N_{h} / N$, $(h=1,2,3, \ldots, L)$ are unknown. In these conditions double sampling for stratification is used. Procedure for double sampling for stratification is given below:

(a) A first phase sample $S$ of size $n^{\prime}$, is drawn using simple random sampling without replacement and only auxiliary variate $x$ is observed.

\footnotetext{
${ }^{1}$ Corresponding author: Research Scholar in S.S. in Statistics, Vikram University, Ujjain-456010, India.

E-mail: hilalstat@gmail.com
}

Published 31 May 2015 / journal homepage: http://csam.or.kr

(C) 2015 The Korean Statistical Society, and Korean International Statistical Society. All rights reserved. 
(b) The sample $S^{\prime}$ is stratified into $L$ strata on the basis of auxiliary variable $x$. Let $n_{h}^{\prime}$ be the number of units in $h^{\text {th }}$ stratum $(h=1,2, \ldots, L)$ such that $\sum_{h=1}^{L} n_{h}^{\prime}=n^{\prime}$.

(c) From each $n_{h}^{\prime}$ units, a sample of size $n_{h}=v_{h} n_{h}^{\prime}$ is drawn, where $0<v_{h}<1$ is the predetermined probability of selecting a sample of size $n_{h}$ from strata of size $n_{h}^{\prime}$ and it constitutes a sample $S$ of size $n=\Sigma_{h=1}^{L} n_{h}$. In sample $S$ both study variate $y$ and auxiliary variate $x$ are observed. Let $y$ and $x$ be the study vairate and the auxiliary vairate respectively then we define

- $\bar{x}_{d s}=\Sigma_{h=1}^{L} w_{h} \bar{x}_{h}:$ Unbiased estimator of population mean $\bar{X}$ in second phase or double sampling mean of the auxiliary variate $x$.

- $\bar{y}_{d s}=\Sigma_{h=1}^{L} w_{h} \bar{y}_{h}:$ Unbiased estimator of population mean $\bar{Y}$ in second phase or double sampling mean of the study variate $y$.

- $\bar{z}_{d s}=\Sigma_{h=1}^{L} w_{h} \bar{z}_{h}$ : Unbiased estimator of population mean $\bar{Z}$ in second phase or double sampling mean of the auxiliary variate $z$.

- $\bar{x}_{h}=\frac{1}{n_{h}} \sum_{i=1}^{n_{h}} x_{h i}$ : Mean of the second phase sample taken from $h^{\text {th }}$ stratum for the auxiliary variate $x$.

- $\bar{y}_{h}=\frac{1}{n_{h}} \sum_{i=1}^{n_{h}} y_{h i}$ : Mean of the second phase sample taken from $h^{\text {th }}$ stratum for the study variate $y$.

- $\bar{z}_{h}=\frac{1}{n_{h}} \sum_{i=1}^{n_{h}} z_{h i}:$ Mean of the second phase sample taken from $h^{\text {th }}$ stratum for the auxiliary variate $z$.

- $\bar{X}_{h}=\frac{1}{N_{h}} \Sigma_{i=1}^{N_{h}} x_{h i}: h^{\text {th }}$ stratum mean for the auxiliary variate $x$.

- $\bar{Y}_{h}=\frac{1}{N_{h}} \sum_{i=1}^{N_{h}} y_{h i}: h^{\text {th }}$ stratum mean for the study variate $y$.

- $\bar{Z}_{h}=\frac{1}{N_{h}} \Sigma_{i=1}^{N_{h}} z_{h i}: h^{\text {th }}$ stratum mean for the auxiliary variate $z$.

- $\bar{X}=\frac{1}{N} \Sigma_{h=1}^{L} \Sigma_{i=1}^{N_{h}} x_{h i}$ : Population mean of the auxiliary variate $x$.

- $\bar{Y}=\frac{1}{N} \Sigma_{h=1}^{L} \Sigma_{i=1}^{N_{h}} y_{h i}$ : Population mean of the study variate $y$.

- $\bar{Z}=\frac{1}{N} \Sigma_{h=1}^{L} \Sigma_{i=1}^{N_{h}} z_{h i}$ : Population mean of the auxiliary variate $z$.

- $S_{x}^{2}=\frac{1}{N-1} \sum_{h=1}^{L} \sum_{i=1}^{N_{h}}\left(x_{h i}-\bar{X}_{h}\right)^{2}$ : Population mean square of the auxiliary variate $x$.

- $S_{y}^{2}=\frac{1}{N-1} \sum_{h=1}^{L} \sum_{i=1}^{N_{h}}\left(y_{h i}-\bar{Y}_{h}\right)^{2}$ : Population mean square of the study variate $y$.

- $S_{z}^{2}=\frac{1}{N-1} \sum_{h=1}^{L} \sum_{i=1}^{N_{h}}\left(z_{h i}-\bar{Z}_{h}\right)^{2}$ : Population mean square of the auxiliary variate $z$.

- $S_{x h}^{2}=\frac{1}{N_{h}-1} \sum_{i=1}^{N_{h}}\left(x_{h i}-\bar{X}_{h}\right)^{2}: h^{\text {th }}$ stratum mean square of the auxiliary variate $x$.

- $S_{y h}^{2}=\frac{1}{N_{h}-1} \sum_{i=1}^{N_{h}}\left(y_{h i}-\bar{Y}_{h}\right)^{2}: h^{\text {th }}$ stratum mean square of the study variate $y$.

- $S_{z h}^{2}=\frac{1}{N_{h}-1} \sum_{i=1}^{N_{h}}\left(z_{h i}-\bar{Z}_{h}\right)^{2}: h^{\text {th }}$ stratum mean square of the auxiliary variate $z$.

- $\bar{x}_{h}^{\prime}=\frac{1}{n_{h}^{\prime}} \sum_{i=1}^{n_{h}^{\prime}} x_{h i}$ : First phase sample mean of the of $h^{\text {th }}$ stratum for the auxiliary variate $x$.

- $\bar{z}_{h}^{\prime}=\frac{1}{n_{h}^{\prime}} \sum_{i=1}^{n_{h}^{\prime}} z_{h i}$ : First phase sample mean of the of $h^{\text {th }}$ stratum for the auxiliary variate $z$.

- $f=\frac{n^{\prime}}{N}$ : First phase sampling fraction.

- $w_{h}=\frac{n_{h}^{\prime}}{n^{\prime}}: h^{\text {th }}$ stratum weight in the first phase sample. 
- $n=\Sigma_{h=1}^{L} n_{h}:$ Size of the sample $S^{\prime}$

- $\bar{x}^{\prime}=\sum_{h=1}^{L} \bar{x}_{h}^{\prime}$ : First phase sample mean of the of $h^{\text {th }}$ stratum for the auxiliary variate $x$.

- $\bar{z}^{\prime}=\Sigma_{h=1}^{L} \bar{z}_{h}^{\prime}$ : First phase sample mean of the of $h^{\text {th }}$ stratum for the auxiliary variate $z$.

The variance of usual unbiased estimator $\bar{y}_{d s}$ in double sampling for stratification is given by

$$
V\left(\bar{y}_{d s}\right)=S_{y}^{2}\left(\frac{1-f}{n^{\prime}}\right)+\frac{1}{n^{\prime}} \sum_{h=1}^{L} W_{h}\left(\frac{1}{v_{h}}-1\right) S_{y h}^{2} .
$$

Ige and Tripathi (1987) studied classical ratio and product type estimators in double sampling for stratification as

$$
\hat{\bar{Y}}_{R}^{d s}=\bar{y}_{d s}\left(\frac{\bar{x}^{\prime}}{\bar{x}_{d s}}\right)
$$

and

$$
\hat{\bar{Y}}_{P}^{d s}=\bar{y}_{d s}\left(\frac{\bar{z}_{d s}}{\bar{z}^{\prime}}\right),
$$

where $z$ is an auxiliary variate negatively correlated with the study variate $y$.

The biases and mean square errors of estimators $\hat{\bar{Y}}_{R}^{d s}$ and $\hat{\bar{Y}}_{P}^{d s}$, upto the first degree of approximation, are obtained as

$$
\begin{aligned}
B\left(\hat{\bar{Y}}_{R}^{d s}\right) & =\frac{1}{n^{\prime}} \sum_{h=1}^{L} W_{h}\left(\frac{1}{v_{h}}-1\right) \frac{1}{\bar{X}}\left(R_{1} S_{y h}^{2}-S_{y x h}\right), \\
B\left(\hat{\bar{Y}}_{P}^{d s}\right) & =\frac{1}{\bar{Z}} \frac{1}{n^{\prime}} \sum_{h=1}^{L} W_{h}\left(\frac{1}{v_{h}}-1\right) S_{y z h}, \\
\operatorname{MSE}\left(\hat{\bar{Y}}_{R}^{d s}\right) & =S_{y}^{2}\left(\frac{1-f}{n^{\prime}}\right)+\frac{1}{n^{\prime}} \sum_{h=1}^{L} W_{h}\left(\frac{1}{v_{h}}-1\right)\left[S_{y h}^{2}+R_{1}^{2} S_{y h}^{2}-2 R_{1} S_{y x h}\right]
\end{aligned}
$$

and

$$
\operatorname{MSE}\left(\hat{\bar{Y}}_{P}^{d s}\right)=S_{y}^{2}\left(\frac{1-f}{n^{\prime}}\right)+\frac{1}{n^{\prime}} \sum_{h=1}^{L} W_{h}\left(\frac{1}{v_{h}}-1\right)\left[S_{y h}^{2}+R_{2}^{2} S_{z h}^{2}+2 R_{2} S_{y z h}\right]
$$

where $W_{h}=N_{h} / N, R_{1}=\bar{Y} / \bar{X}$ and $R_{2}=\bar{Y} / \bar{Z}$.

Assuming that the information on the two auxiliary variates $x$ and $z$ are known, Singh (1967) suggested the following ratio-cum-product estimator for finite population mean of the study variate in simple random sampling as

$$
\hat{\bar{Y}}=\bar{y}\left(\frac{\bar{X}}{\bar{x}}\right)\left(\frac{\bar{z}}{\bar{Z}}\right)
$$

Koyuncu and Kadilar (2009) proposed Singh (1967) estimators in stratified random sampling as

$$
\hat{\bar{Y}}_{s t}=\bar{y}_{s t}\left(\frac{\bar{X}}{\bar{x}_{s t}}\right)\left(\frac{\bar{z}_{s t}}{\bar{Z}}\right) \text {. }
$$




\section{Proposed Estimator}

Motivated by Koyuncu and Kadilar (2009), generalized ratio-cum-product type estimators in double sampling for stratification is proposed as

$$
\hat{\bar{Y}}_{d s}^{(\alpha, \beta)}=\bar{y}_{d s}\left(\frac{\bar{x}^{\prime}}{\bar{x}_{d s}}\right)^{\alpha}\left(\frac{\bar{z}_{d s}}{\bar{z}^{\prime}}\right)^{\beta},
$$

where $\alpha$ and $\beta$ are suitably chosen scalar, and can be determined such that the mean squared error of $\hat{\bar{Y}}_{d s}^{(\alpha, \beta)}$ is minimum.

It is to be noted that:

(i) for $(\alpha=0, \beta=0)$ in $(2.1), \hat{\bar{Y}}_{d s}^{(\alpha, \beta)}$ reduces to

$$
\hat{\bar{Y}}_{d s}^{(0,0)}=\bar{y}_{d s}
$$

which is usual unbiased estimator of population mean $\bar{Y}$.

(ii) for $(\alpha=1, \beta=0)$ in (2.1), $\hat{\bar{Y}}_{d s}^{(\alpha, \beta)}$ reduces to the ratio type estimator suggested by Ige and Tripathi (1987) as

$$
\hat{\bar{Y}}_{d s}^{(1,0)}=\bar{y}_{d s}\left(\frac{\bar{x}^{\prime}}{\bar{x}_{d s}}\right) .
$$

(iii) for $(\alpha=0, \beta=1)$ in $(2.1), \hat{\bar{Y}}_{d s}^{(\alpha, \beta)}$ reduces to the product type estimator suggested by Ige and Tripathi (1987) as

$$
\hat{\bar{Y}}_{d s}^{(0,1)}=\bar{y}_{d s}\left(\frac{\bar{z}_{d s}}{\bar{z}^{\prime}}\right)
$$

(iv) for $(\alpha=1, \beta=1)$ in $(2.1), \hat{\bar{Y}}_{d s}^{(\alpha, \beta)}$ reduces to

$$
\hat{\bar{Y}}_{d s}^{(1,1)}=\bar{y}_{d s}\left(\frac{\bar{x}^{\prime}}{\bar{x}_{d s}}\right)\left(\frac{\bar{z}_{d s}}{\bar{z}^{\prime}}\right) .
$$

To obtain the bias and mean square error of the proposed estimator $\hat{\bar{Y}}_{d s}^{(\alpha, \beta)}$ we write

$$
\bar{y}_{d s}=\bar{Y}\left(1+e_{0}\right), \quad \bar{x}_{d s}=\bar{X}\left(1+e_{1}\right), \quad \bar{x}^{\prime}=\bar{X}\left(1+e_{1}^{\prime}\right), \quad \bar{z}_{d s}=\bar{Z}\left(1+e_{2}\right) \quad \text { and } \quad \bar{z}^{\prime}=\bar{Z}\left(1+e_{2}^{\prime}\right)
$$

such that

$$
\begin{aligned}
& E\left(e_{0}\right)=E\left(e_{1}\right)=E\left(e_{1}^{\prime}\right)=E\left(e_{2}\right)=E\left(e_{2}^{\prime}\right)=0, \\
& \left.E\left(e_{0}^{2}\right)=\frac{1}{\bar{Y}^{2}}\left[S_{y}^{2}\left(\frac{1-f}{n^{\prime}}\right)+\frac{1}{n^{\prime}} \sum_{h=1}^{L} W_{h}\left(\frac{1}{v_{h}}-1\right) S_{y h}^{2}\right)\right], \\
& \left.E\left(e_{1}^{2}\right)=\frac{1}{\bar{X}^{2}}\left[S_{x}^{2}\left(\frac{1-f}{n^{\prime}}\right)+\frac{1}{n^{\prime}} \sum_{h=1}^{L} W_{h}\left(\frac{1}{v_{h}}-1\right) S_{x h}^{2}\right)\right], \\
& \left.E\left(e_{2}^{2}\right)=\frac{1}{\bar{Z}^{2}}\left[S_{z}^{2}\left(\frac{1-f}{n^{\prime}}\right)+\frac{1}{n^{\prime}} \sum_{h=1}^{L} W_{h}\left(\frac{1}{v_{h}}-1\right) S_{z h}^{2}\right)\right],
\end{aligned}
$$




$$
\begin{aligned}
& \left.E\left(e_{0} e_{1}\right)=\frac{1}{\bar{Y} \bar{X}}\left[S_{y x}\left(\frac{1-f}{n^{\prime}}\right)+\frac{1}{n^{\prime}} \sum_{h=1}^{L} W_{h}\left(\frac{1}{v_{h}}-1\right) S_{y x h}\right)\right], \\
& \left.E\left(e_{0} e_{2}\right)=\frac{1}{\bar{Y} \bar{Z}}\left[S_{y z}\left(\frac{1-f}{n^{\prime}}\right)+\frac{1}{n^{\prime}} \sum_{h=1}^{L} W_{h}\left(\frac{1}{v_{h}}-1\right) S_{y z h}\right)\right], \\
& \left.E\left(e_{1} e_{2}\right)=\frac{1}{\bar{X} \bar{Z}}\left[S_{x z}\left(\frac{1-f}{n^{\prime}}\right)+\frac{1}{n^{\prime}} \sum_{h=1}^{L} W_{h}\left(\frac{1}{v_{h}}-1\right) S_{x z h}\right)\right], \\
& E\left(e_{0} e_{1}^{\prime}\right)=\frac{1}{\bar{Y} \bar{X}}\left(\frac{1-f}{n^{\prime}}\right) S_{y x h}, \\
& E\left(e_{1}^{\prime 2}\right)=\frac{1}{\bar{X}^{2}} S_{x}^{2}\left(\frac{1-f}{n^{\prime}}\right), \\
& E\left(e_{1} e_{1}^{\prime}\right)=\frac{1}{\bar{X}}\left(\frac{1-f}{n^{\prime}}\right) S_{x}^{2} .
\end{aligned}
$$

Now expressing the proposed estimator $\hat{\bar{Y}}_{d s}^{(\alpha, \beta)}$ in terms of $e$ 's, we have

$$
\begin{aligned}
\left(\hat{\bar{Y}}_{d s}^{(\alpha, \beta)}-\bar{Y}\right)= & \bar{Y}\left[e_{0}+\alpha\left(e_{1}^{\prime}-e_{1}-e_{0} e_{1}+e_{0} e_{1}^{\prime}\right)+\beta\left(e_{2}-e_{2}^{\prime}+e_{0} e_{2}-e_{0} e_{2}^{\prime}\right)\right. \\
& +\frac{\alpha(\alpha+1)}{2} e_{1}^{2}+\frac{\alpha(\alpha-1)}{2} e_{1}^{\prime 2}+\frac{\beta(\beta+1)}{2} e_{2}^{\prime 2}+\frac{\beta(\beta-1)}{2} e_{2}^{2}-\alpha^{2} e_{1} e_{1}^{\prime}-\beta^{2} e_{2} e_{2}^{\prime} \\
& \left.+\alpha \beta\left(e_{1} e_{2}^{\prime}-e_{1} e_{2}-e_{1}^{\prime} e_{2}^{\prime}+e_{1}^{\prime} e_{2}\right)\right] .
\end{aligned}
$$

Taking expectations on both sides of (2.6), bias of the proposed estimator $\hat{\bar{Y}}_{d s}^{(\alpha, \beta)}$ to the first degree of approximation is obtained as

$$
\begin{aligned}
B\left(\hat{\bar{Y}}_{d s}^{(\alpha, \beta)}\right)= & \bar{Y}\left[\frac{\alpha(\alpha+1)}{2} \frac{1}{\bar{X}^{2}}\left\{S_{x}^{2}\left(\frac{1-f}{n^{\prime}}\right)+\frac{1}{n^{\prime}} \sum_{h=1}^{L} W_{h}\left(\frac{1}{v_{h}}-1\right) S_{x h}^{2}\right\}+\frac{\alpha(\alpha-1)}{2} \frac{S_{x}^{2}}{\bar{X}^{2}}\left(\frac{1-f}{n^{\prime}}\right)\right. \\
& +\frac{\beta(\beta+1)}{2} \frac{S_{z}^{2}}{\bar{Z}^{2}}\left(\frac{1-f}{n^{\prime}}\right)+\frac{\beta(\beta-1)}{2} \frac{1}{\bar{Z}^{2}}\left\{S_{z}^{2}\left(\frac{1-f}{n^{\prime}}\right)+\frac{1}{n^{\prime}} \sum_{h=1}^{L} W_{h}\left(\frac{1}{v_{h}}-1\right) S_{z h}^{2}\right\} \\
& \left.-\alpha^{2} \frac{S_{x}^{2}}{\bar{X}^{2}}\left(\frac{1-f}{n^{\prime}}\right)-\beta^{2} \frac{S_{z}^{2}}{\bar{Z}^{2}}\left(\frac{1-f}{n^{\prime}}\right)-\frac{1}{n^{\prime}} \sum_{h=1}^{L} W_{h}\left(\frac{1}{v_{h}}-1\right)\left\{\frac{\alpha \beta}{\bar{X} \bar{Z}} S_{x z h}-\frac{\beta}{\bar{Y} \bar{Z}} S_{y z h}+\frac{\alpha}{\bar{X} \bar{Y}} S_{y x h}\right\}\right] .
\end{aligned}
$$

Squaring both sides of (2.6) and then taking expectations, we get the mean square error of the proposed estimator $\hat{\bar{Y}}_{d s}^{(\alpha, \beta)}$ upto the first degree of approximation as

$$
\begin{aligned}
\operatorname{MSE}\left(\hat{\bar{Y}}_{d s}^{(\alpha, \beta)}\right)= & S_{y}^{2}\left(\frac{1-f}{n^{\prime}}\right)+\frac{1}{n^{\prime}} \sum_{h=1}^{L} W_{h}\left(\frac{1}{v_{h}}-1\right)\left[S_{y h}^{2}+\alpha^{2} R_{1}^{2} S_{x h}^{2}+\beta^{2} R_{2}^{2} S_{z h}^{2}-2 \alpha R_{1} S_{y x h}\right. \\
& \left.+2 \beta R_{2} S_{y z h}-2 \alpha \beta R_{1} R_{2} S_{x z h}\right] .
\end{aligned}
$$

Equation (2.8) can also be written as

$$
\operatorname{MSE}\left(\hat{\bar{Y}}_{d s}^{(\alpha, \beta)}\right)=A+B+\alpha^{2} R_{1}^{2} C+\beta^{2} R_{2}^{2} D-2\left(\alpha R_{1} E-\beta R_{2} F+\alpha \beta R_{1} R_{2} G\right)
$$


where

$$
\begin{aligned}
& A=S_{y}^{2}\left(\frac{1-f}{n^{\prime}}\right) \\
& B=\frac{1}{n^{\prime}} \sum_{h=1}^{L} W_{h}\left(\frac{1}{v_{h}}-1\right) S_{y h}^{2}, \\
& C=\frac{1}{n^{\prime}} \sum_{h=1}^{L} W_{h}\left(\frac{1}{v_{h}}-1\right) S_{x h}^{2}, \\
& D=\frac{1}{n^{\prime}} \sum_{h=1}^{L} W_{h}\left(\frac{1}{v_{h}}-1\right) S_{z h}^{2}, \\
& E=\frac{1}{n^{\prime}} \sum_{h=1}^{L} W_{h}\left(\frac{1}{v_{h}}-1\right) S_{y x h}, \\
& F=\frac{1}{n^{\prime}} \sum_{h=1}^{L} W_{h}\left(\frac{1}{v_{h}}-1\right) S_{y z h}, \\
& G=\frac{1}{n^{\prime}} \sum_{h=1}^{L} W_{h}\left(\frac{1}{v_{h}}-1\right) S_{x z h} .
\end{aligned}
$$

The mean square error of the proposed estimator $\hat{\bar{Y}}_{d s}^{(\alpha, \beta)}$ is minimum when

$$
\alpha=\frac{(E D-G F)}{R_{1}\left(C D-G^{2}\right)}
$$

and

$$
\beta=\frac{(E G-F C)}{R_{2}\left(C D-G^{2}\right)} .
$$

Thus the resulting minimum mean square error of the proposed estimator $\hat{\bar{Y}}_{d s}^{(\alpha, \beta)}$ is

$$
\operatorname{MSE}_{\text {Min }}\left(\hat{\bar{Y}}_{d s}^{(\alpha, \beta)}\right)=\gamma\left(1-\frac{\mu}{\gamma}\right),
$$

where $\gamma=A+B, \mu=\left(D E^{2}+C F^{2}-2 E F G\right) /\left(C D-G^{2}\right)$ and $A, B, C, D, E, F$ and $G$ have their meanings.

\section{Study on the Particular Member $\hat{\bar{Y}}_{d s}^{(1,1)}$ of the Generalized Ratio-Cum-Product Type Estimator $\hat{\bar{Y}}_{d s}^{(\alpha, \beta)}$}

To illustrate our result, we have study a particular member of the proposed generalized ratio-cumproduct type estimator $\hat{\bar{Y}}_{d s}^{(\alpha, \beta)}$ given in (2.4) as

$$
\hat{\bar{Y}}_{d s}^{(1,1)}=\bar{y}_{d s}\left(\frac{\bar{x}^{\prime}}{\bar{x}_{d s}}\right)\left(\frac{\bar{z}_{d s}}{\bar{z}^{\prime}}\right) .
$$


Putting $(\alpha=1, \beta=1)$ in (2.7) and (2.8), the bias and mean square error of the estimator $\hat{\bar{Y}}_{d s}^{(1,1)}$ are obtained as

$$
B\left(\hat{\bar{Y}}_{d s}^{(1,1)}\right)=\frac{1}{n^{\prime}} \sum_{h=1}^{L} W_{h}\left(\frac{1}{v_{h}}-1\right)\left[\frac{1}{\bar{X}}\left(R_{1} S_{x h}^{2}-S_{y x h}\right)+\frac{1}{\bar{Z}}\left(S_{y z h}-R_{1} S_{x z h}\right)\right]
$$

and

$$
\begin{aligned}
\operatorname{MSE}\left(\hat{\bar{Y}}_{d s}^{(1,1)}\right)= & S_{y}^{2}\left(\frac{1-f}{n^{\prime}}\right)+\frac{1}{n^{\prime}} \sum_{h=1}^{L} W_{h}\left(\frac{1}{v_{h}}-1\right)\left[S_{y h}^{2}+R_{1}^{2} S_{x h}^{2}+R_{2}^{2} S_{z h}^{2}\right. \\
& \left.-2 R_{1} S_{y x h}+2 R_{2} S_{y z h}-2 R_{1} R_{2} S_{x z h}\right] .
\end{aligned}
$$

From (1.1), (1.6), (1.7) and (3.3), it is observed that the estimator $\hat{\bar{Y}}_{d s}^{(1,1)}$ would be more efficient than

(i) usual unbiased estimator $\bar{y}_{d s}$ if

$$
\sum_{h=1}^{L} W_{h}\left(\frac{1}{v_{h}}-1\right)\left[R_{1}^{2} S_{x h}^{2}+R_{2}^{2} S_{z h}^{2}-2 R_{1} S_{y x h}+2 R_{2} S_{y z h}-2 R_{1} R_{2} S_{x z h}\right]<0 .
$$

(ii) Ige and Tripathi (1987) ratio type estimator $\hat{\bar{Y}}_{R}^{d s}$ if

$$
\sum_{h=1}^{L} W_{h}\left(\frac{1}{v_{h}}-1\right)\left[R_{2} S_{z h}^{2}+2 S_{y z h}\right]<2 \sum_{h=1}^{L} W_{h}\left(\frac{1}{v_{h}}-1\right) R_{1} S_{x z h} .
$$

(iii) Ige and Tripathi (1987) ratio type estimator $\hat{\bar{Y}}_{P}^{d s}$ if

$$
\sum_{h=1}^{L} W_{h}\left(\frac{1}{v_{h}}-1\right)\left[R_{1} S_{x h}^{2}-2 S_{y x h}\right]<2 \sum_{h=1}^{L} W_{h}\left(\frac{1}{v_{h}}-1\right) R_{2} S_{x z h}
$$

Expressions (3.4), (3.5) and (3.6) are the conditions under which the estimator $\hat{\bar{Y}}_{d s}^{(1,1)}$, a particular member of the proposed generalized ratio-cum-product type estimator $\hat{\bar{Y}}^{(\alpha, \beta)}$, has less mean square error as compared to the mean square error of the other considered estimators.

Remark 1. It is to be noted that upto the first degree of approximation, the biases and mean square errors of the estimators $\bar{y}_{d s}, \hat{\bar{Y}}_{R}^{d s}, \hat{\bar{Y}}_{P}^{d s}$, and $\hat{\bar{Y}}^{(1,1)}$ are obtained from (2.7) and (2.8), by replacing the values $(\alpha, \beta)$ by $(0,0),(1,0),(0,1)$ and $(1,1)$, respectively.

\section{Efficiency Comparisons}

Comparisons of(1.1), (1.6), (1.7), (2.12) and (3.3), show that

(i) $\operatorname{MSE}\left(\hat{\bar{Y}}_{d s}^{\left(\alpha_{0}, \beta_{0}\right)}\right)<V\left(\bar{y}_{d s}\right)$ if

$$
E^{2} D+F^{2} C-2 E F G>0
$$


Table 1: Population I

\begin{tabular}{c|cc}
\hline \hline Estimators & Stratum I & Stratum II \\
\hline$n_{h}$ & 2 & 2 \\
$n_{h}^{\prime}$ & 4 & 4 \\
$N_{h}$ & 10 & 3.67 \\
$\bar{Y}_{h}$ & 1.70 & 289.14 \\
$\bar{X}_{h}$ & 10.41 & 80.67 \\
$\bar{Z}_{h}$ & 6.32 & 1.14 \\
$S_{y h}$ & 0.50 & 11.61 \\
$S_{x h}$ & 3.53 & 10.82 \\
$S_{z h}$ & 1.19 & 144.88 \\
$S_{y x h}$ & 1.61 & -7.05 \\
$S_{y z h}$ & -0.06 & -92.02 \\
$S_{x z h}^{2}$ & 1.38 & 2.21 \\
$S_{y}^{2}$ & & \\
\hline
\end{tabular}

Source by Tailor et al. (2014). $y=$ output; $x=$ fixed capital; $z=$ number of workers.

Table 2: Population II

\begin{tabular}{c|cc}
\hline \hline Estimators & Stratum I & Stratum II \\
\hline$n_{h}$ & 2 & 2 \\
$n_{h}^{\prime}$ & 4 & 5 \\
$N_{h}$ & 5 & 315.60 \\
$\bar{Y}_{h}$ & 1925.80 & 333.80 \\
$\bar{X}_{h}$ & 214.40 & 60.60 \\
$\bar{Z}_{h}$ & 51.80 & 340.38 \\
$S_{y h}$ & 615.92 & 66.35 \\
$S_{x h}$ & 74.87 & 4.84 \\
$S_{z h}$ & 0.75 & 22356.50 \\
$S_{y x h}$ & 39360.68 & 1536.24 \\
$S_{y z h}$ & 411.16 & 287.92 \\
$S_{x z h}^{2}$ & 30.08 & \\
$S_{y}^{2}$ & 668351.00 & \\
\hline
\end{tabular}

Source by Murthy (1967). $y=$ output; $x=$ fixed capital; $z=$ number of workers.

(ii) $\operatorname{MSE}\left(\hat{\bar{Y}}_{d s}^{\left(\alpha_{0}, \beta_{0}\right)}\right)<\operatorname{MSE}\left(\hat{\bar{Y}}_{R}^{d s}\right)$ if

$$
R_{1}^{2} C^{2} D-R_{1}^{2} C G^{2}-2 R_{1} C D^{2}+2 R_{1} D G^{2}+D E^{2}+C F^{2}-2 E F G>0 .
$$

(iii) $\operatorname{MSE}\left(\hat{\bar{Y}}_{d s}^{\left(\alpha_{0}, \beta_{0}\right)}\right)<\operatorname{MSE}\left(\hat{\bar{Y}}_{P}^{d s}\right)$ if

$$
R_{2}^{2} C D^{2}-R_{2}^{2} D G^{2}+2 R_{2} F G^{2}+2 R_{2} F C D+D E^{2}+C F^{2}-2 E F G>0 .
$$

(iv) $\operatorname{MSE}\left(\hat{\bar{Y}}_{d s}^{\left(\alpha_{0}, \beta_{0}\right)}\right)<\operatorname{MSE}\left(\hat{\bar{Y}}_{d s}^{(1,1)}\right)$ if

$$
\left(R_{1}^{2} C+R_{2}^{2} D-2 R_{1} E+2 R_{2} F-2 R_{1} R_{2} G\right)\left(C D-G^{2}\right)+D E^{2}+C F^{2}-2 E F G>0 .
$$

\section{Empirical Study}

Two population data sets are considered to exhibit the performance of the proposed estimators in comparison to other considered estimators. Tables 1 and 2 provides the description of the populations. 
Table 3: Percent relative efficiencies of $\bar{y}_{d s}, \hat{\bar{Y}}_{R}^{d s}, \hat{\bar{Y}}_{P}^{d s}, \hat{\bar{Y}}_{d s}^{(1,1)}$ and $\hat{\bar{Y}}^{\left(\alpha_{0}, \beta_{0}\right)}$ with respect to $\bar{y}_{d s}$

\begin{tabular}{c|cc}
\hline \hline Estimators & Population I & Population II \\
\hline $\bar{y}_{d s}$ & 100.00 & 100.00 \\
$\hat{\bar{Y}}_{R}^{d s}$ & 144.99 & 160.69 \\
$\hat{\bar{Y}}_{P}^{d s}$ & 111.86 & 77.73 \\
$\hat{\bar{Y}}_{d s}^{(1,1)}$ & 158.09 & 178.24 \\
$\hat{\bar{Y}}^{\left(\alpha_{0}, \beta_{0}\right)}$ & 203.15 & 199.80 \\
\hline \hline
\end{tabular}

Table 4: Exhibition of theoretical conditions given in (3.4), (3.5) and (3.6) empirically

\begin{tabular}{l|cc}
\multicolumn{1}{c}{ Conditions } & Population I & Population II \\
\hline \hline $\operatorname{MSE}\left(\hat{\bar{Y}}_{d s}^{(1,1)}\right)<V\left(\bar{y}_{d s}\right)$ if & & \\
$\sum_{h=1}^{L} W_{h}\left(\frac{1}{v_{h}}-1\right)\left[R_{1}^{2} S_{x h}^{2}+R_{2}^{2} S_{z h}^{2}-2 R_{1} S_{y x h}+2 R_{2} S_{y z h}-2 R_{1} R_{2} S_{x z h}\right]<0$ & $-0.55<0$ & $-167370<0$ \\
\hline $\operatorname{MSE}\left(\hat{\bar{Y}}_{d s}^{(1,1)}\right)<\operatorname{MSE}\left(\hat{\bar{Y}}_{R}^{d s}\right)$ if & & \\
$\sum_{h=1}^{L} W_{h}\left(\frac{1}{v_{h}}-1\right)\left[R_{2} S_{z h}^{2}+2 S_{y z h}\right]<2$ & $-2.58<-1.21$ & $2485.6<2997.9$ \\
$\sum_{h=1}^{L} W_{h}\left(\frac{1}{v_{h}}-1\right) R_{1} S_{x z h}$ & & \\
\hline $\operatorname{MSE}\left(\hat{\bar{Y}}_{d s}^{(1, I)}\right) \operatorname{MSE}\left(\hat{\bar{Y}}_{P}^{d s}\right)$ if & & \\
$\sum_{h=1}^{L} W_{h}\left(\frac{1}{v_{h}}-1\right)\left[R_{1} S_{x h}^{2}-2 S_{y x h}\right]<2$ & $-26.23<-4.1$ & $-15701<14621.9$ \\
$\sum_{h=1}^{L} W_{h}\left(\frac{1}{v_{h}}-1\right) R_{2} S_{x z h}$ & \\
\hline \hline
\end{tabular}

\section{Conclusion}

Table 3 indicates that the proposed generalized ratio-cum-product type estimator is more efficient than $\bar{y}_{d s}, \hat{\bar{Y}}_{R}^{d s}, \hat{\bar{Y}}_{P}^{d s}$ and $\hat{\bar{Y}}^{(1,1)}$ in both population data sets. Section 4 provides the conditions under which the proposed generalized ratio-cum-product type estimator $\hat{\bar{Y}}^{(\alpha, \beta)}$ has less mean square error compared to the mean square errors of other considered estimators.

Expressions (3.4), (3.5) and (3.6) deals with the theoretical efficiency comparisons of the particular member $\hat{\bar{Y}}^{(1,1)}$ with usual unbiased estimator $\bar{y}_{d s}$ and Ige and Tripathi (1987) estimators $\hat{\bar{Y}}_{R}^{d s}$ and $\hat{\bar{Y}}_{P}^{d s}$. Conditions under which the estimator $\hat{\bar{Y}}^{(1,1)}$ has less mean square error than other considered estimators are calculated empirically and tabulated in Table 4. Table 3 shows that the estimator $\hat{\bar{Y}}^{(1,1)}$, a particular member of the proposed generalized ratio-cum-product type estimator $\hat{\bar{Y}}^{(\alpha, \beta)}$ has higher percent relative efficiency compared to usual unbiased estimator $\bar{y}_{d s}$ and Ige and Tripathi (1987) estimators $\hat{\bar{Y}}_{R}^{d s}$ and $\hat{\bar{Y}}_{P}^{d s}$. Therefore the estimator $\hat{\bar{Y}}^{(1,1)}$ is recommended for use in practice if the conditions (3.4), (3.5) and (3.6) are satisfied.

\section{Discussion}

In this paper, a generalized ratio-cum-product type estimator has been proposed. Usual unbiased estimator, Ige and Tripathi (1987) estimator and a particular member of the proposed estimator are identified as the members of the proposed estimator. More estimators can be generated by choosing a suitable values of $\alpha$ and $\beta$. It is important to note that conditions under which proposed estimator (including particular identified member) are more efficient than the other obtained considered estimators. As per the limitations point of view, these estimators perform better if the obtained conditions are satisfied. One more limitation is the simulation study that shows the performance of estimators in connection with the behavior of known parameters. In future studies, some more estimators in the line of proposed estimator can be developed which perform better in most of the situations. 


\section{Acknowledgment}

The authors are thankful to the editor in chief and two learned reviewers for their valuable suggestions that helped a lot to improve this paper.

\section{References}

Cochran, W. G. (1940). The estimation of the yields of cereal experiments by sampling for the ratio of grain to total produce, Journal of Agricultural Science, 30, 262-275.

Ige, A. F. and Tripathi, T. P. (1987). On double sampling for stratification and use of auxiliary information, Journal of the Indian Society of Agricultural Statistics, 39, 191-201.

Koyuncu, N. and Kadilar, C. (2009). Family of estimators of population mean using two auxiliary variable in stratified random sampling, Communication in Statistics - Theory and Methods, 38, 2398-2417.

Murthy, M. N. (1967). Sampling Theory and Methods, Statistical Publishing Society, Calcutta.

Robson, D. S. (1957). Applications of multivariate polykays to the theory of unbiased radio-type estimation, Journal of the American Statistical Association, 52, 511-522.

Singh, M. P. (1967). Radio cum product method of estimation, Metrika, 12, 34-42.

Singh, H. P. and Vishwakarma, G. K. (2007). A general procedure for estimating the mean using double sampling for stratification, Model Assisted Statistics and Applications, 2, 225-237.

Tailor, R., Chouhan, S. and Kim, J. M. (2014). Ratio and product type exponential estimators of population mean in double sampling for stratification, Communications for Statistical Applications and Methods, 21, 1-9.

Tailor, R. and Lone, H. A. (2014). Radio-cum-product estimator of finite population mean in double sampling for stratification, Journal of Reliability and Statistical Studies, 7, 93-101. 